\title{
Evaluation of Cancer-Associated Fibroblasts Markers in Hepatocellular Carcinoma
}

\author{
GHADA A. ABD EL-FATTAH, M.D.; RASHA M. ABDRABUH, M.D. and RANIA G. ROSHDY, M.D.
}

The Department of Pathology, Faculty of Medicine, Benha University

\begin{abstract}
Background: Cancer associated fibroblasts (CAFs) are main components of tumor microenvirnoment. They act an essential role in tumor progression, invasion and unresponsiveness to chemotherapy.
\end{abstract}

Aim of Study: This work aims to study the relation of CAFs with hepatocellular carcinoma (HCC) developement and progression.

Material and Methods: This is an uncontrolled retrospective study carried upon 30 cases of HCC. Cases were collected from archives of Pathology Department and Early Cancer Detection Unit (ECDU), Faculty of Medicine, Benha University, and from International Medical Center during the years 2010-2015. Podoplanin (D2-40), alpha smooth muscle actin $(\alpha-S M A)$ and CD31 immunohistochemichal staining were performed and evaluated for each case.

Results: Both D2-40 and $\alpha$-SMA showed different distributions and levels of expression. D2-40 positivity was localized to the peri-tumoral area, while $\alpha$-SMA was detected in both intra- and peritumoral areas. Expression of both markers was not statistically related to each other $(p>0.05)$. D2-40 was positive in $73.3 \%$ of casesand $\alpha$-SMA showed high expressionin $60 \%$ of cases. Both positive D2-40 and high $\alpha$-SMA showed positive relations to tumor grade $(p<0.01$ for both markers), associated cirrhosis ( $p<0.05$ for both markers), lymphatic ( $p<0.05$ for both markers) and vascular invasion $(p<0.05$ and $p<0.01$ respectively). Also positive relations were reported between positivity of D2-40 and lymph node metastases $(p<0.05)$. Neoangiogenesis was estimated by positivity to CD31 and Microvessel density (MVD) was calculated. Lymphatic vessel density (LVD) was also estimated by using positivity to D2-40. Both mean MVD and meanLVD were significantly increased in relation to positive D2-40 expression ( $p<0.01$ and $p<0.05$ respectively). Also, the higher $\alpha$-SMA expression, the higher the means of both MVD and LVD. Theses also were highly statistically significant relations $(p<0.01$ for both $)$.

Conclusion: Cancer associated fibroblasts have a possible role in development and progression of HCC. This could be implemented in new therapeutic strategies for HCC.

Correspondence to: Dr. Ghada A. Abd El-Fattah, The Department of Pathology, Faculty of Medicine, Benha University
Key Words: Cancer associated fibroblasts (CAFs) - Hepatocellular carcinoma (HCC) - Podoplanin (D2-40) - Alpha smmoth muscle actin (a-SMA) - CD31Microvessel density (MVD) - Lymphatic vessel density (LVD).

\section{Introduction}

WORLDWIDE, hepatocellular carcinoma (HCC) is considered to be the sixth most common malignant neoplasm and the third leading cause of deaths from cancer [1]. In Egypt, A registry done by AinShams Faculty of Medicine reported that HCC represents about $2.4 \%$ of total malignancy and $12.8 \%$ of malignant digestive system tumors. Liver cirrhosis was reported in $77 \%$ of HCCs and positivity for $\mathrm{HBV}$ and/or $\mathrm{HCV}$ was present in $90 \%$ of them [2]

In different solid tumors (including HCC), not only malignant cells are responsible for tumorigenesis and tumor progression, but also tumor stroma is involved and is considered animportant participant and regulator. An interaction between tumor cells and stroma forms what is known as tumorhost microenvironment [3]. Tumor microenviroment acts a pivotal role in tumor growth and progression, metastasis, in angiogensis and resistance to chemotherapy [4] .

A major element of cancer stroma, is activated fibroblasts, which are known as the cancerassociated fibroblasts (CAFs) [5]

CAFs show myofibroblast- like characteristics and express a variety of proteins as $\alpha$-SMA, platelets-derived growth factor (PDGF) Platelets-derived growth factor (PDGF), fibronectin, integrin $\alpha-11$ and podoplanin (D2-40). The expression level of these proteins vary in different CAFs. None of these markers are unique for CAFs [6]. 
Alpha smooth muscle actin ( $\alpha$-SMA) is an actin isoform kwown to be expressed in the vascular smooth-muscle cells. This protein also has an important role in the process of fibrogenesis [7].

Podoplanin (D2-40), another marker for CAFs, is a transmembrane glycoprotein of mucin-type [8] . Different studies identified podoplanin (D240) expression of CAFs as an indicatorof poor survival as in breast, esophageal, lung tumors ,or a favorable prognostic indicatoras in colorectal cancer [9].

However, in a study made on breast carcinoma, detection of lymphatic invasion was observed using D2-40 which was also found to be a good marker to discriminate lymphatic invasion from blood vessel invasion. Lymphovascular invasion is associated with occurrence of nodal metastasis and recurrence of the tumor. As peer the same study, CD 31 was found to be aneffective marker to detectblood vessel invasion [10]

This work aims to evaluate the relation of CAFs (detected by D2-40 and $\alpha$-SMA) with HCC development and progression Looking forward to find new treatment options for HCC as it has become a major health problem in Egypt.

\section{Material and Methods}

This is an uncontrolled retrospective study carried upon 30 specimens of formalin-fixed, paraffin-embedded hepatocellular carcinoma. None of the patients had received preoperative chemotherapy. Cases were obtained from archieves of Pathology Departement and Early Cancer Detection Unit (ECDU), Faculty of Medicine, Benha University, and from International Medical Center during the years 2010-2015.Cases were graded into well, moderate and poorly differentiated HCC [11]. Tumor cases were classified into 2 groups; tumors less than $5 \mathrm{~cm}$ in size and tumors larger than $5 \mathrm{~cm}$ in size [12]. Staging were done according to AJCC 2018 [13] . Paraffin-embedded tissue sections were prepared from obtained specimens. To confirm diagnosis and grading; two pathologists reviewed Hematoxylin and Eosin sections.

\section{Immunohistochemical study:}

Three formalin-fixed, paraffin-embedded, 4micron tissue sections were prepared for each case. Immunohistochemical detection of D2-40, $\alpha$-SMA and CD31 antigens was done (Table 1). Streptavidin-biotin technique was used following the manufacturer's instructions (Neomarker, LABVISION, USA, CA 94538-7310). The sections were visualizedwith $0.02 \%$ diaminobenzidine (DAB) solution. Finally sections were counterstained with hematoxylin then dehydrated and mounted. A negative control was used for each marker, by omitting the primary antibody and replacing it with normal rabbit serum IgG.

\section{Immunohistochemical interpretation:}

D2-40 showed cytoplasmic staining of the lymphatic vessels'endothelial cells. A structure with a central lumen, lined by endothelial cells, was taken as positive vessel. Lymphatic vessel density (LVD) was detected using D2-40 expression in endothelial cells lining lymphatic vessels newly formed by the tumor. Lymphatic vessel invasion was classified into low and high regarding the calculated mean LVD in this work $[9,12]$

The stromal staining of cancer associated fibroblasts by D2-40 and $\alpha$-SMA, was interpreted according to Choi, et al., [14]

Microvessel density (MVD) were detected both peri- and intratumoral using CD31 expression in endothelial cells lining these vessels (Fig. 1C). Microvessel invasion was subgrouped into low and high according to the calculated mean MVD. $[9,15]$.

Table (1): Antibodies studied in this work.

\begin{tabular}{lllllll}
\hline Antibody & Source & Cat. No. & Dilution & Incubation period & Positive control & $\begin{array}{c}\text { Staining } \\
\text { pattern }\end{array}$ \\
\hline D2-40 & GeneTex, USA & GTX31231 & $1: 50$ & 30 minutes at room temp & Benign tonsillar tissue & Cytoplasmic \\
a-SMA & $\begin{array}{c}\text { Lab vision/ } \\
\text { Thermoscientific }\end{array}$ & A1 5764 & $1: 200$ & 60 minutesatroom temp & $\begin{array}{c}\text { Vascular smooth muscle } \\
\text { cells }\end{array}$ & Cytoplasmic \\
& USA & & & Benign tonsillar tissue & Cytoplasmic \\
CD31 & $\begin{array}{l}\text { DAKO, Glostrup, } \\
\text { Denmark }\end{array}$ & $\begin{array}{c}\text { Mouse anti } \\
\text { human, } \\
\text { clone JC70A }\end{array}$ & $1: 50$ & Overnight at $4^{\circ} \mathrm{C}$ & & \\
\hline
\end{tabular}


Statistical analysis: The obtained data were analyzed using SPSS version 16 software (SpssInc, Chicago, ILL Company). The Pearson correlation test was performed to determine the association of clinico-pathological data with D2-40, $\alpha$-SMA and CD31 expression. The value of $(p<0.05)$ was considered significant and level of $(p<0.01)$ was considered as highly significant.

\section{Results}

This work was carried upon 30 cases of HCC. Nineteen patients were males and 11 were females. The ages of patients ranged from 49 to 70 years with mean age of 58.8 years. There were $5(16.7 \%)$ casesof well differentiated HCC, 14 (46.6\%) cases ofmoderately differentiated HCC and 11 (36.7\%) cases of poorly differentiated HCC. Among the studied tumor cases, there were $18(60 \%)$ HCCs associated with cirrhosis. As regarding TNM staging, $6(20 \%)$ cases were stage I, $5(16.7 \%)$ cases were stage II, $8(26.6 \%)$ cases were stage III and $11(36.7 \%)$ cases were stage IV.
Cancer associated fibroblasts (CAFs) appeared as stromal elongated spindlecells having brown cytoplasmic staining. Immunohistochemical detectionof CAFs by both D2-40 and $\alpha$-SMA showed different distributions and levels of expression. Twenty-two (73.3\%) cases showed positive D240 and $18(60 \%)$ cases showed high $\alpha$-SMA expression. D2-40 positivity was localized to the peri-tumoral area (Fig. 1A) while $\alpha$-SMA was detected in both intra- and peri-tumoral areas (Fig. 1B). Expression of both markers was not statistically related to each other $(p>0.05)$.

Both positive D2-40 and high $\alpha$-SMA showed positive relations to tumor grade $(p<0.01$ for both markers), associated cirrhosis ( $p<0.05$ for both markers), lymphatic ( $p<0.05$ for both markers) and vascular invasion ( $p<0.05$ and $p<0.01$ respectively). Also positive correlation were reported between positivity of D2-40 and LN metastases $(p<0.05)$ (Table 2).

Table (2): Relation of CAFs markers to the studied clinico-pathological parameters.

\begin{tabular}{|c|c|c|c|c|c|c|}
\hline \multirow{2}{*}{$\begin{array}{l}\text { Clinicopathological } \\
\text { parameters }\end{array}$} & \multicolumn{2}{|c|}{ D2-40 } & \multirow{2}{*}{$p$-value } & \multicolumn{2}{|c|}{$\alpha$-SMA } & \multirow{2}{*}{$p$-value } \\
\hline & Negative & Positive & & Low & High & \\
\hline \multicolumn{7}{|l|}{ Tumor grade: } \\
\hline Grade1 & $3(10 \%)$ & $2 \quad(6.7 \%)$ & $<0.01 * *$ & $5 \quad(16.7 \%)$ & $0 \quad(0 \%)$ & $<0.01 * *$ \\
\hline Grade2 & $5(16.7 \%)$ & $9 \quad(30 \%)$ & & $7 \quad(23.3 \%)$ & $7 \quad(23.3 \%)$ & \\
\hline Grade 3 & $0(0 \%)$ & $11(36.6 \%)$ & & $0 \quad(0 \%)$ & $11(36.6 \%)$ & \\
\hline \multicolumn{7}{|l|}{ Associated cirrhosis: } \\
\hline Absent & $6(20 \%)$ & $6 \quad(20 \%)$ & $<0.05^{*}$ & $8 \quad(26.7 \%)$ & $4 \quad(13.3 \%)$ & $<0.05^{*}$ \\
\hline Present & $2(6.7 \%)$ & $16(53.3 \%)$ & & $4 \quad(13.3 \%)$ & $14(46.7 \%)$ & \\
\hline \multicolumn{7}{|l|}{ Tumor size: } \\
\hline$<5 \mathrm{~cm}$ & $4(13.3 \%)$ & $13(43.4 \%)$ & $>0.05$ & $8 \quad(26.7 \%)$ & $9 \quad(30 \%)$ & $>0.05$ \\
\hline$\geq 5 \mathrm{~cm}$ & $4(13.3 \%)$ & $9 \quad(30 \%)$ & & $4 \quad(13.3 \%)$ & $9 \quad(30 \%)$ & \\
\hline \multicolumn{7}{|l|}{$N:$} \\
\hline Negative & $8(26.7 \%)$ & $11(36.6 \%)$ & $<0.05^{*}$ & $8 \quad(26.7 \%)$ & $11(36.6 \%)$ & $>0.05$ \\
\hline Positive & $0(0 \%)$ & $11(36.6 \%)$ & & $4 \quad(13.3 \%)$ & $7 \quad(23.3 \%)$ & \\
\hline \multicolumn{7}{|l|}{ M: } \\
\hline Negative & $8(26.7 \%)$ & $15(50 \%)$ & $>0.05$ & $10(\%)$ & $13(43.3 \%)$ & $>0.05$ \\
\hline Positive & $0(0 \%)$ & $7 \quad(23.3 \%)$ & & $2(6.7 \%)$ & $5 \quad(16.7 \%)$ & \\
\hline \multicolumn{7}{|l|}{ Tumor stage: } \\
\hline I & $3(10 \%)$ & $3 \quad(10 \%)$ & $>0.05$ & $4 \quad(13.3 \%)$ & $2(6.7 \%)$ & $>0.05$ \\
\hline II & $1(3.3 \%)$ & $4 \quad(13.3 \%)$ & & $1 \quad(3.3 \%)$ & $4 \quad(13.3 \%)$ & \\
\hline III & $4(13.3 \%)$ & $4 \quad(13.3 \%)$ & & $3 \quad(10 \%)$ & $5 \quad(16.7 \%)$ & \\
\hline IV & $0(0 \%)$ & $11(36.6 \%)$ & & $4 \quad(13.3 \%)$ & $7 \quad(23.3 \%)$ & \\
\hline \multicolumn{7}{|l|}{ Vascular invasion: } \\
\hline Low & $6(20 \%)$ & $7 \quad(23.3 \%)$ & $<0.05^{*}$ & $9 \quad(30 \%)$ & $4 \quad(13.3 \%)$ & $<0.01 * *$ \\
\hline High & $2(6.7 \%)$ & $15(50 \%)$ & & $3(10 \%)$ & $14(46.7 \%)$ & \\
\hline \multicolumn{7}{|l|}{ Lymphatic invasion: } \\
\hline Low & $6(20 \%)$ & $7 \quad(23.3 \%)$ & $<0.05^{*}$ & $8 \quad(26.7 \%)$ & $5 \quad(16.7 \%)$ & $<0.05^{*}$ \\
\hline High & $2(6.7 \%)$ & $15(50 \%)$ & & $4 \quad(13.3 \%)$ & $13(43.3 \%)$ & \\
\hline Total & 8 & 22 & & 12 & 18 & \\
\hline
\end{tabular}


Mean LVD calculated for studied cases was $34.70 \pm$ 8.655, while mean MVD was 47.61 \pm 10.263 . Both mean MVD and mean LVD were significantly increased with positive D2-40 expression $(p<0.01$ and $p<0.05$ respectively). On the other hand, the higher $\alpha$-SMA expression, the higher the means of both MVD \& LVD. Theses also were highly statistically significant relations ( $p<0.01$ for both) (Table 3$)$.

Table (3): Relations between MVD, LVD and CAFs markers.

\begin{tabular}{|c|c|c|c|c|c|c|}
\hline CAFs markers & Mean MVD & SD & $p$-value & Mean LVD & SD & $p$-value \\
\hline \multicolumn{7}{|l|}{$D 2-40:$} \\
\hline Negative & 38.15 & \pm 10.955 & $<0.01 * *$ & 28.62 & \pm 6.501 & $<0.05^{*}$ \\
\hline Positive & 51.05 & \pm 7.681 & & 36.91 & \pm 8.377 & \\
\hline \multicolumn{7}{|l|}{$\alpha-S M A:$} \\
\hline Low & 41.77 & \pm 10.401 & $<0.01 * *$ & 29.25 & \pm 8.895 & $<0.01 * *$ \\
\hline High & 51.50 & \pm 8.340 & & 38.33 & \pm 6.453 & \\
\hline
\end{tabular}

a-SMA: Alpha smooth muscle actin. $\quad$ *: Significant. $\quad$ **: Highly significant.
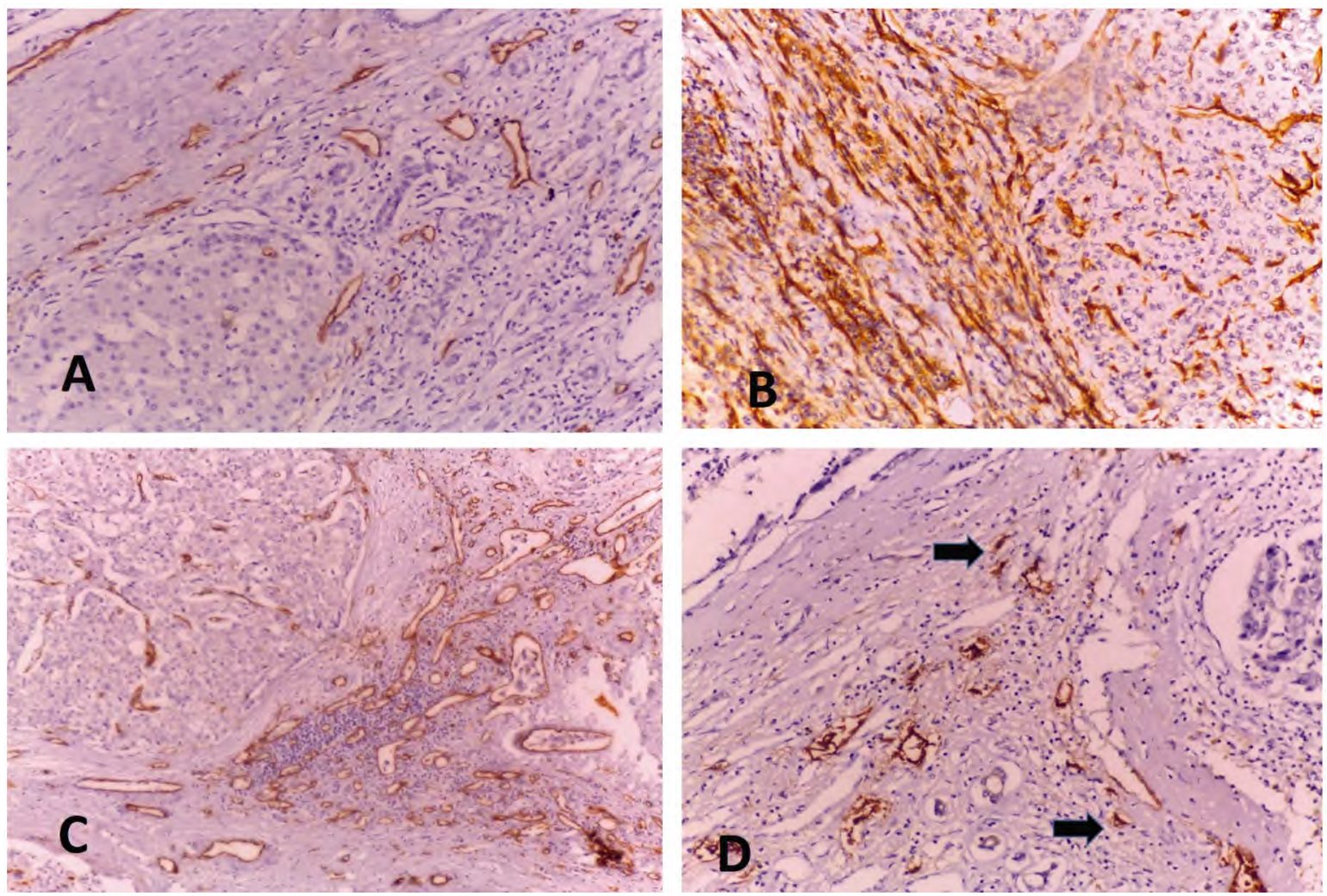

Fig. (1):

(A): Positive D2-40 peritumoral expression. ABC x 200.

(B): High $\alpha$-SMA both peri- and intratumoral expression. ABC x 200.

(C): Neo-angiogenesis detected by CD31 positivity. ABC x 100.

(D): Elongated spindledCAFs labeled by D2-40 (black arrows). ABC x 400.

\section{Discussion}

Hepatocellular carcinoma is well identified to be one of the most aggressive types of neoplasms which doesn't have effective therapeutic lines. Most cases develop in the setting of chronic liver injury, hepatitis, and hepatic fibrosis [16]

In different types of tumors, CAFs may originate from the activation of quiescent fibroblasts, epi- thelial-mesenchymal transition and may also be derived from mesenchymal stem cells of bone marrow. In HCC, they originate mainly from hepatic stellate cells [17].

In this retrospective work, thirty cases of hepatocellular carcinomas ofdifferent stages and grades are studied immunohistochemcially for $\alpha$-SMA, D2-40 and CD31 expressions. 
Although both $0 \tau$-SMA and D2-40 are considered markers for activated CAFs, our study revealed different patterns of expression as regard location and intensity for both of them as. D2-40 positive $\mathrm{CAFs}$ were localized to the peri-tumoral area while those stained by ot-SMA were detected in both peri-tumoral and intra-tumoral areas. Also no statistically significant correlation was found between them $(p>0.05)$. This result is confirmed by a study done by Rei et al., [9] who compared different markers of CAFs in advanced colorectal carcinoma denoting that CAFs markers differ in their ability to detect fibroblasts.

Our study also revealed a statistically significant correlation between positive expression of D240 and high expression of ot -SMA in CAFs with high tumor grade ( $p<0.01$ for both) and associated cirrhosis- a well-known precancerous condition for HCC ( $<<0.05$ for both). Many previous invivo and invitro studies have revealed three different mechanisms by which CAFs increase malignant cell proliferation; first by secreting numerous factors and inflammatory cytokines as IL- $10 \tau$, IL6 , CXCL- 8 chemokine, cyclooxygenase 2 (COX2 ), CCL20, Kindlin-2 which activates $[3$-integrins which are transmembrane receptors promote cellextracellular matrix adhesion; second via induce epithelial-mesenchymal transition; and lastly by cross talking with immune cells and inhibiting immune surveillance $[\mathbf{1 6 , 2 1}]$

This positive correlation between tumor grade and CAFs markers was also seen in studies done by Kadotaetal., [18], Patil et al., [19], Kim et al., [20] on non-small cell carcinoma of the lung, oral squamous cell carcinoma and squamous cell carcinoma of the head and neck respectively.

This pattern of positive expression of CAFs to D2-40 and their high expression of or-SMA insures the malignant potential of both markers in HCC and their role in carcinogenesis and progression. Kalluri, et al., [17] in his study found that activation of CAFs promotes tumorigenesis through production of inflammatory and growth factors.

In the current study, there was a statistically significant correlation between vascular invasion and both positive expression of D2-40 and high expression of $0 \tau$-SMA in CAFs $(p<0.05$ and $<0.01$ respectively) and between them and lymphatic invasion $(p<0.05)$. These results could be explained by the results of Boire et al., [22] who found that activated CAFs which express either D2-40 or $0 \tau-$ SMA can promote invasion of tumor cells by secreting proteases as matrix metalloproteinase that degrade extracellular matrix. More importantly D2-40 increases the ability of fibroblasts to promote motility and invasiveness by the amplification of RhoA which has a role in cytoskeletal dynamics, cell motion and other cellular functions [23].

Several previous studies have shown that lymphangiogenesis has a direct relation to tumor growth, progression and its invasion [24]. In this work we used immunohistochemical staining of D2-40 to measure LVD. There was a positive significant correlation between LVD and lymph node metastasis with high or-SMA $(p<0.01)$ and positive D2-40 expression in CAFs $(p<0.05$ for each). This was in agreement with Takagi et al., [25], Lin et al., [26] and Hu et al., [27], in their studies on oral squamous cell carcinoma, lung carcinoma and bladder carcinoma respectively.

Mean microvesseldensity (MVD) and determination of number of newly-formed vessels in tumor tissue is of great importance to assess neoangiogenesis. This has been detected by several techniques such as immunohistochemistryand others. Here we used immunohistochemical staining of CD3 1 to measure MVD. In this study we found a statistically significant correlation between MVD and positive expression of D2-40 and high expression of o $\tau$-SMA in CAFs. Taura, et al., [28] previously have concluded that CAFs secrete many angiogenic factors mainly VEGF, PDGF, angiopoietin- 1 , angiopoietin-2 and vasohibin which play an important role in neoangiogenesis.

Direct positive relations between CAFs markers expression and lymph node metastasis, vascular invasion, MVD and LVD have pointed to their predictive rolein poor prognosis of $\mathrm{HCC}$.

\section{Conclusion:}

This study demonstrated thepossiblerole of CAFs in development, progression and poor prognosis of HCC. It also gives the possibility to the future planning and using of their markersas a target therapy in HCC.

Recommendation: Further researches are recommended to explore the therapeutic importance of CAFs markers and their actions on this problematic neoplasm. Another study is recommended to detect the expression of CAF in different grades of cirrhosis in order to exploretheir role in malignant transformation.

\section{Conflict of interest:}

No conflict of interest. 


\section{References}

1- FORNER A., LIOVET J.M. and BRUIX J.: Hepatocellular carcinoma. Lancet, 379: 1245-1255, 2012.

2- HELA T.A., SALMAN M.I. and EZZ-ELARAB S.S.: Malignant digestive system tumors. In: Pathology-Based Cancer Registry 2001-2010. Ain Shams Faculty of Medicine, Cairo, Egypt, Chapter 5, p 26-50, 2015.

3- KALLURI R.: Basement membranes: Structure, assembly androle in tumour angiogenesis. Nat. Rev. Cancer, 3: 422433, 2003.

4- SUN Q., ZHANG B. and HU Q.: The impact of cancerassociated fibroblasts on majorhallmarks of pancreatic cancer. Theranostics, 8 (18): 5072-5087, 2018.

5- LEE J.S., YOO J.E., KIM H., et al.: Tumor stroma with senescence-associated secretory phenotype in steatohepatitic hepatocellular carcinoma. PLoSOne., 12 (3): e0171922, 2017.

6- CHERNG S., YOUNG J. and MA H.: Alpha smooth Muscle Actin (a-SMA). The Journal of American Science, 4 (4), 2008.

7- CHUAYSRI C., THUWAJIT P., PAUPAIROJ A., et al.: Alpha-smooth muscle actin -positive fibroblasts promote biliary cell proliferation and correlate with poor survival in cholangiocarcinoma. Oncol. Rep., 21: 957 969, 2009.

8- UGORSKI M., DZIEGIEL P. and SUCHANSKI J.: Podoplanin - a small glycoprotein with many faces. Am. J. Cancer Res., 6: 370-386, 2016.

9- NISHISHITA R., MOROHASHI S., SEINO H., et al.: Expression of cancer-associated fibroblasts markers in advanced colorectal cancer. Oncol. Lett., 15 (5): 6195 6202,2018

10- DILEEP and PRASAD P.H.: Use of Immunomarkers D240 and CD31 in Detection of Lymphovascular Invasion in Breast Carcinoma. JMSCR, (6): 07, 2018.

11- World Health Organization Classification of Tumours by InternationalAgency for Research on Cancer: WHO Classification of Tumours of theDigestive System: Volume 3. 4th Revised ed. Lyon: International Agency for Research on Cancer, 2010.

12- CIOCA A., CEAUSU A.R., RAICA M., et al.: The multifaceted role of podoplanin expression in hepatocellular carcinoma. European Journal of Histochemistry, 61: 2707, 2017.

13- AMIN M., EDGE S., GREENE F., et al.: AJCC Cancer Staging Manual, 8 th Edition, 2018.

14- CHOI S.Y., SUNG R., LEE S.J., et al.: Podoplanin, $\alpha$ Smooth Muscle Actin or S 100A4 Expressing CancerAssociated Fibroblasts Are Associated with Different Prognosis in Colorectal Cancers. J. Korean Med. Sci., 28: 1293-1301, 2013.
15- WANG D., STOCKARD C.R., HARKINS L., et al.: Immunohistochemistry in evaluation of neovascularization in tumor xenografts. Biotech. Histochem., 83 (0): 179189, 2008.

16- BAGLIERI J., DAVID A., BRENNER and TATIANA KISSELEVA: The role of fibrosis and liver-associated fibroblasts in the pathogenesis of hepatocellular carcinoma. Int. J. Mol. Sci., 20: 1723, 2019.

17- KALLURI R.: The biology and function of fibroblasts in cancer. Nat. Rev. Cancer, 16: 582-98, 2016.

18- KATDOTA K., HAUNG C.L., LIU D., et al.: The clinical significance of the tumor cell D2-40 immunoreactivity in non -small lung cancer. Lung Cancer, 70: 88-93, 2010.

19- PATIL A., PATIL K., TUPSAKHARE S., et al.: Evaluation of podoplanin in oral leukoplakia and oral squamous cell carcinoma.Scientifica, 2015: 135298, 2015.

20- KIM H., RHA K., SHIM G.A., et al.: Podoplanin is involved in the prognosis of head and neck squamous cell carcinoma through interaction with VEGF-C. Oncol. Rep., 34: 833-42, 2015.

21- LOTTI R., MARCONI A., TRUZZI F., et al.: A previously unreported function of $\beta$ (1)B integrin isoform in caspase8 -dependent integrin-mediated keratinocyte death. J. Invest Dermatol., 2; 130 (11): 2569-77, 2010.

22- BOIRE A., COVIC L., AGARWAL A., et al.: PAR1 is a matrix metalloprotease-1 receptor that promotes invasion and tumorigenesis of breast cancer cells. Cell., 120 (3): 303-3 13, 2005

23- ITO S., ISHII G., HOSHINO A., et al.: Tumor promoting effect of podoplanin-positive fibroblasts is mediated by enhanced RhoA activity. Biochem. Biophys Res. Commun., 422 (1): 194-199, 2012.

24- THELEN A., JONAS S., BENCKERT C., et al.: Tumor -associated lymphangiogenesis correlates with prognosis after resection of human hepatocellular carcinoma. Ann. Surg. Oncol., 16: 1222-30, 2009.

25- TAKAGI S., OH-HARA T., SATA S., et al.: Expression of Aggrus/podoplanin in bladder cancer and its role in pulmonary metastasis. Int. J. Cancer, 134: 2605-14, 2014.

26- LIN N.N., WANG P., ZHAO D., et al.: Significance of oral cancer associated fibroblasts in angiogenesis, lymphangiogenesis, and tumor invasion in oral squamous cell carcinoma. J. Oral Pathol. Med., 46: 21-30, 2017.

27- HU G., ZHONG K., CHEN W., et al.: Podoplanin -postive cancer associated fibroblasts predict poor prognosis in lung cancer patients. Dovepress, Volume 2018, p. 5607 $5619,2018$.

28- TAURA K., De MINICIS S., SEKI E.,et al.: Hepatic stellate cells secrete angiopoitin 1 that induces angiogenesis in liver fibrosis. Gastroenterology, 135: 1729-1738, 2008 . 


\section{تقييم ظهور دلالات الخلايا الليفية المرتبطة

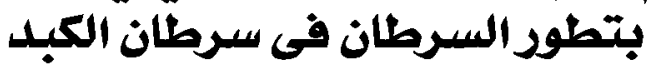

يعتبر سرطان الكبد (HCC) سادس أكثر الأودام الخبيثة شيوعاً فى جميع أنحاء العالم والسبب الرئيسى الثالث لوفيات السرطان وقد

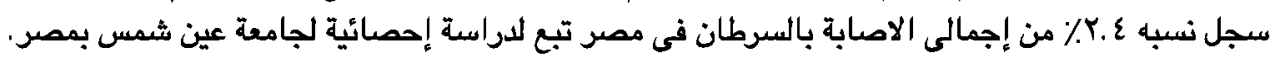

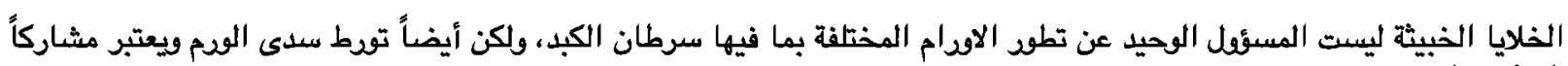

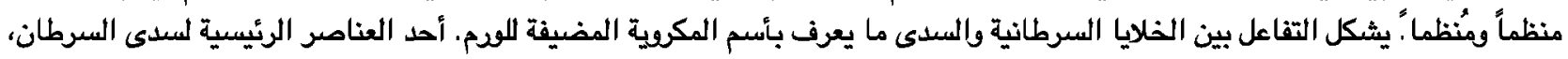

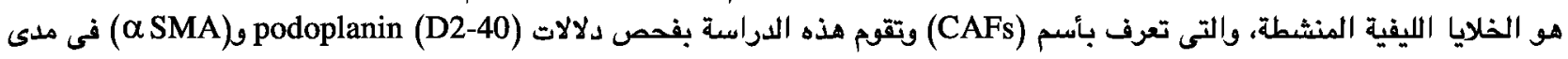
تتطو سرطان الكبد.

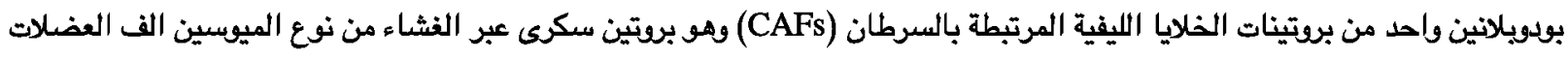

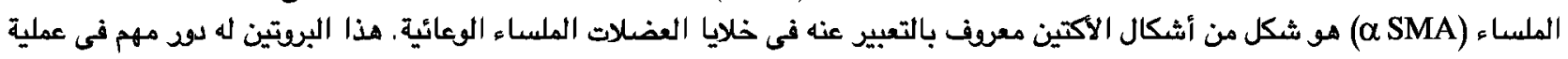
الموم الليفى.

وقد أشارت الدراسة إلى وجود علاقات إيجابية مباشرة بين تعبير علامات CAF ودم خبيث العقدة الليمفاوية، والغزو الوعائى، MVD

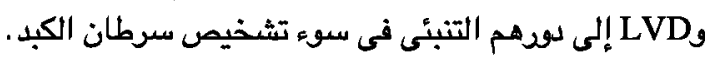

ومن ثم اثبت اليود المحتمل لا CAFs في التمية والتقدم وسوء التشخيص من سرطان الكبد. كما أنه يعطى إمكانية لتخطيط في المستقبل

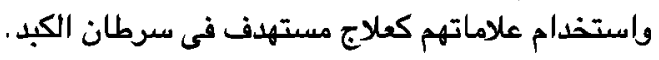

